\title{
Prototypicality of arguments and conformity to ingroup norms
}

\author{
DAAN VAN KNIPPENBERG* and \\ HENK WILKE \\ University of Leiden, The Netherlands
}

\section{Abstract}

Social categorization is claimed to elicit a tendency to conform to ingroup norms, which may result in attitude change after exposure to information on the opinions of other ingroup members. It was hypothesized that the degree to which arguments represented ingroup norms, i.e., were prototypical, would affect their potential influence on attitudes, such that prototypical arguments would be perceived as being of higher quality and would elicit more attitude change. Moreover, prototypical arguments were expected to elicit more argument elaboration. Two experiments were designed to test these predictions. In Experiment 1 subjects were exposed to both a set of pro and a set of contra arguments, while one of the sets was allegedly prototypical of ingroup attitudes. In Experiment 2 subjects were exposed to either prototypical or a-prototypical pro or contra arguments allegedly originating from in- or outgroup. In both studies conformity to ingroup norms was observed. In addition, prototypical ingroup arguments elicited higher quality ratings in the first study. Indications of higher elaboration of prototypical ingroup arguments were found.

\section{INTRODUCTION}

During the last few years increasing attention has been turned to social influence processes as affected by social categorizations (e.g. Mackie, 1986; Turner, Hogg, Oakes, Reicher and Wetherell, 1987; Hogg and Turner, 1987; Abrams and Hogg, 1990; Wilder, 1990). These authors assert that social influence processes like conformity and group polarization are mediated by group identification. Extending earlier theorizing on conformity (e.g. Sherif, 1936; Festinger, Schachter and Back, 1950; Deutsch and Gerard, 1955; Kiesler and Kiesler, 1969), Turner et al. (1987) stress the intergroup context in which conformity processes take place. Turner $e t$ al. contend

Addressee for correspondence: Daan Van Knippenberg, Department of Social and Organizational Psychology, Wassenaarseweg 52, 2333 AK Leiden, The Netherlands.

* Netherlands organization for scientific research (NWO) is gratefully acknowledged for funding this project (PSYCHON-grant 560-270-034). 
in their self-categorizational approach to social influence that identification with a social group to social influence that identification with a social group will elicit a tendency to conform to ingroup norms. This tendency may affect group members' attitudes, as illustrated by e.g. Mackie (1986; Mackie and Cooper, 1984) and Abrams, Wetherell, Cochrane, Hogg and Turner (1990). Both Mackie and Abrams et al. assert that after exposure to information about attitudes held by others, subsequent influence of this information on own attitudes is affected by group membership of the source and receiver. For example, Mackie (1986) presented subjects with arguments allegedly generated in a group discussion. The source group was either an ingroup or an outgroup. Subjects could derive the source group opinion towards the attitude topic from the arguments presented. Subjects' attitudes turned out to be more affected by exposure to ingroup arguments than by exposure to outgroup arguments, presumably as a consequence of the hypothesized tendency to conform to ingroup norms.

The mediating role of group norms is further illustrated in a study by Turner, Wetherell and Hogg (1989). Subjects participated in a group discussion on choice dilemmas involving more or less risky courses of action. Prior to the discussion either a risky or a cautious group norm was experimentally induced. Subjects in allegedly risky groups displayed more risky decisions than subjects in groups that were presumably cautious, i.e. subjects tended to behave according to the group norm. Turner et al. maintain that this result is due to a tendency to perceive arguments which are in line with the group norm (i.e. pro risk arguments in case of a risky norm and pro caution arguments in case of a cautious norm) as stronger and more valid than arguments opposing the group norm. That is, arguments representing the group norm ('prototypical' arguments) are more persuasive. However, since the group discussion was not under experimental control in the Turner et al. study, further testing of this hypothesis seems necessary.

\section{EXPERIMENT 1}

In the present study subjects were exposed both to arguments supporting and opposing the use of university entrance exams. These arguments were allegedly generated by own group and were presented as being either prototypical or a-prototypical of the normative group opinion. We hypothesized subjects would conform to the group norm, that is, to the position advocated by the prototypical arguments (hypothesis 1a). Furthermore, as contended by Turner et al. (1989), we expected prototypical arguments would be perceived to be of higher quality than a-prototypical arguments (hypothesis 1b).

Recently, it has been proposed that social categorization will not only elicit a tendency to conform to ingroup norms, but will also result in a higher motivation to elaborate ingroup communication as compared to outgroup communication (Mackie, Worth and Asuncion, 1990; Van Knippenberg and Wilke, 1991). Since one's primary interest supposedly lies with one's own group and group norms, information expressing ingroup attitudes was hypothesized to elicit a higher motivation to elaborate. Both Mackie $e t$ al. and Van Knippenberg and Wilke presented subjects with strong or weak arguments allegedly originating from in- or outgroup. Argument quality is assumed to affect attitudes to a greater extent under conditions of high 
message elaboration ( $c f$. Chaiken, 1980, 1987; Petty and Cacioppo, 1981, 1986). When a message is intensively scrutinized and the message content is carefully evaluated, strong arguments may result in substantial attitude change while weak arguments will not. This differential influence is less apparent under conditions of low elaboration. both Mackie $e t$ al. and Van Knippenberg and Wilke found attitudes to be affected by argument quality after exposure to ingroup arguments, but not after exposure to outgroup arguments. This result was taken to be indicative of more elaboration of the ingroup message.

In the studies by Mackie $e t$ al. and Van Knippenberg and Wilke arguments could be assumed to be prototypical of the source group. The very fact that ingroup arguments presumable represented ingroup attitudes may have elicited the apparent higher motivation to elaborate ingroup communication. It is proposed here that when arguments are less prototypical of ingroup norms they will not only elicit less attitude change, but may also result in less message elaboration than prototypical arguments (hypothesis 2). The quality of the presented arguments was varied experimentally in the present study to test this hypothesis. Attitudes were predicted to be affected to a greater extent by the quality of prototypical arguments than by the quality of a-prototypical arguments.

\section{Method}

\section{Overview and design}

Subjects were confronted with two sets of arguments concerning the use of university entrance exams. One set contained arguments supporting entrance exams, the other consisted of arguments opposing the use of these exams. The arguments were allegedly generated by the subjects' own group (psychology undergraduates). Quality of the presented pro and contra arguments was varied independently across conditions. In addition, one of the sets allegedly represented the source position (which was experimentally varied), while the other did not. The order in which the sets were presented was varied over conditions. This yielded a 2(source norm: supporting/ opposing) $\times 2$ (quality of pro arguments: strong/weak) $\times 2$ (quality of contra arguments) $\times 2$ (order of presentation) between-subjects design. The main dependent variables were attitudes assessed on pre- and post-test, cognitive responses to the message and argument recall.

\section{Subjects}

Ninety-five psychology undergraduates ( 28 male, 67 female) from the University of Leiden, the Netherlands, participated in the experiment, receiving Dfl. 10 for their cooperation. Subjects were randomly assigned to experimental conditions. No initial differences in attitudes towards entrance exams, as assessed on the pretest, were found $(M=14.28$, on a 31 -point rating scale with 31 indicating agreement with the use of entrance exams). 


\section{Stimulus material}

The argument sets employed were identical to the ones pretested and used by Van Knippenberg and Wilke (1991, Experiment 2). Four sets of three arguments concerning the use of entrance exams were employed, two sets consisting of strong arguments, one set opposing, one set supporting the use of university entrance exams, and two sets of weak arguments, one opposing, one supporting entrance exams. Argument sets were selected on the basis of a pretest in which strong arguments as compared to weak arguments elicited more positive cognitive responses ( $c f$. Petty and Cacioppo, 1981, 1986, see Procedure below) and more positive evaluations on ratings of argument quality. E.g. 'Entrance exams, being a single measurement, are not representative of a person's abilities' was considered to be a strong argument opposing entrance exams, while e.g. 'It's perfectly normal to prove you're capable of fulfilling necessary requirements' was considered a weak argument supporting the use of these exams.

\section{Procedure}

Pretest attitudes (on 31-point disagree-agree scales) towards a number of issues (including the use of university entrance exams) were assessed when subjects volunteered for participation some weeks prior to the actual experiment.

Subjects attended the experiment in groups of up to eight persons per session. They were placed in individual cubicles containing an Apple Mackintosh computer, which was used to present all experimental stimuli. Subjects were told that a selection was made of arguments concerning the use of entrance exams, allegedly generated in a questionnaire filled out by most of the group members. Subjects were to be presented with two sets of arguments, one set presumably representative of the source opinion, the other set not representative. Sets were explicitly labelled 'representative' and 'not representative'. Subjects were exposed to both a set of pro and a set of contra arguments. Whether the pro or the contra arguments were said to be prototypical of the source opinion was experimentally varied. In addition, the order in which the sets were presented was varied over conditions.

After exposure to the arguments, subjects again expressed their attitudes towards entrance exams (post-test) and completed a thought-listing task ( $c f$. Petty and Cacioppo, 1981, 1986), reporting the thoughts they had while reading the message. Listed thoughts ('cognitive responses') were categorized and rated for their evaluative content by independent judges. The number of message-related cognitive responses may be considered to be indicative of message elaboration. More message elaboration is assumed to result in more message-related responses. Furthermore, when processed systematically, strong arguments elicit positive (favourable to the message content) cognitive responses, while weak arguments elicit negative (unfavourable) responses (cf. Petty and Cacioppo, 1986).

After completing the thought-listing task, subjects rated the quality and the prototypicality of the arguments (7-point scales, higher scores indicating higher quality/ prototypicality) and the perceived source group norm (31-point disagree-agree scale). At the end of the experiment, subjects were requested to write down the presented arguments as they remembered them. Argument recall may be considered to be an additional indication of message elaboration (more elaboration resulting in higher 
recall). After the assessment of argument recall, subjects were debriefed and paid for their participation.

\section{Results and discussion}

Preliminary analyses were performed including the order of argument presentation (pro/contra) as a between-subjects factor in the design. Since no effects of the orderfactor were found, and this factor was not related to any of the hypotheses, this factor was dropped from the design.

\section{Source norm, prototypicality and quality}

The manipulation of the source norm turned out to be successful. When a supportive source norm was induced, the source group was perceived to be supporting the use of university entrance exams $(M=17.35)$, while the source group was assumed to be opposing these exams $(M=10.09)$ when the source norm was opposing the use of entrance exams $(F(1,87)=34.04, p<0.0001)$.

Ratings of the prototypicality of the arguments were analysed by means of ANOVA with the position advocated by the arguments as a within-subjects factor (subjects were confronted with both pro and contra arguments). Analysis indicated that the prototypicality manipulation was successful $(F(1,87)=19.80, p<0.0001)$. When the source group norm was supporting of the use of entrance exams pro arguments were perceived to be more prototypical $(M=4.23)$ than contra arguments $(M=3.56$, $t(47)=2.45, p<0.02$ ), while the reverse was true when the source group was assumed to be opposing the use of entrance exams (pro: $M=3.54$; contra: $M=4.55$, $t(46)=3.89, p<0.0001)$.

Strong contra arguments were perceived to be of higher quality $(M=4.77)$ than weak contra arguments $(M=3.63, F(1,87=20.12, p<0.0001)$. However, no differences in perceived quality were found between strong and weak pro arguments $(F<1)$. This may have been due to the fact that subjects tended to be opposed to the use of entrance exams, which might have elicited an overall negative response to the pro arguments.

To test the prediction that prototypical argument would be perceived to be of higher quality an ANOVA with the position advocated by the arguments as a withinsubjects factor was performed. Analysis revealed a source norm by position of arguments interaction $(F(1,87)=24.06, p<0.0001)$, indicating that contra arguments were perceived to be of higher quality $(M=4.85)$ than pro arguments $(M=3.64$, $t(46)=3.78, p<0.0001$ ) when the source group norm was opposing the use of entrance exams, while contra arguments were rated as being of lower quality $(M=3.54)$ than pro arguments $(M=4.42, t(47)=2.93, p<0.005)$ when the source group was supporting the use of entrance exams. I.e. prototypical arguments were judged to be of higher quality than a-prototypical arguments (hypothesis $1 \mathrm{~b}$ ). No other tests reached significance.

\section{Attitudes}

Post-test attitude scores were analysed by means of ANCOVA with pretest scores as the covariate $(F(1,86)=61.22, p<0.0001$, for the regression). A summary of means 
(adjusted for the pretest) is provided in Table 1. Subjects' attitudes were more opposed to the use of university entrance exams when the source group was assumed to be opposing the use of these exams $(M=11.82)$ than when the source group was supporting the use of entrance exams $(M=15.38,(F(1,86)=7.05, p<0.001))$. I.e. subjects tended to conform to the source group norm ( $c f$. hypothesis 1a). No effects of argument quality were found, an issue which will be addressed below.

Table 1. Post-test attitudes, adjusted for the pretest, Experiment 1

\begin{tabular}{llllll}
\hline & \multicolumn{4}{c}{ Source norm } \\
& \multicolumn{2}{c}{ Supporting } & & Opposing \\
\cline { 2 - 3 } \cline { 5 - 6 } Contra quality & Strong & Weak & & Strong & Weak \\
\hline Pro quality & & & & \\
Strong & 14.85 & 18.67 & & 10.61 & 12.40 \\
Weak & 12.04 & 15.95 & & 12.41 & 11.87 \\
\hline
\end{tabular}

\section{Cognitive responses}

The cognitive responses reported on the thought-listing task were categorized by two independent judges. Target of the response was either categorized as related to the pro arguments, related to the contra arguments, issue-relevant in general or irrelevant. In addition, responses were rated for their evaluative content as positive, negative or neutral. Raters agreed in 74 per cent of the cases on the target of the response and in 75 per cent of the cases on the evaluative content. Mean scores for the two judges were analysed.

To locate possible differences in elaboration of the pro and contra sets, the numbers of cognitive responses explicitly referring to either of the sets were analysed by means of ANOVA with the position advocated by the set as a within-subject factor. Contrary to predictions, prototypical arguments did not elicit more cognitive responses (which would be indicative of higher elaboration) nor did any of the other tests reach significance. Analysis of the general issue-relevant responses yielded no significant results.

Message evaluation, as expressed by the cognitive responses, was operationalized as the difference between the number of positive and the number of negative responses related to each set of arguments. Analysis revealed a main effect of the position advocated by the arguments $(F(1,87)=6.03, p<0.02)$. Contra arguments elicited more positive responses $(M=0.23)$ than pro arguments $(M=-0.13)$. This effect was qualified by the source norm by position interaction $(F(1,87)=7.95, p<0.006)$. When the source group was opposing the use of entrance exams, contra arguments, i.e. prototypical arguments, elicited more positive evaluations $(M=0.47)$ than pro arguments $(M=-0.31, t(46)=4.19, p<0.0001)$, while the evaluation elicited by pro $(M=0.04)$ and contra arguments $(M=-0.01)$ did not differ in case of a supportive source group norm $(t(47)<1)$. The results for the opposing norm conditions are in accordance with the higher ratings of argument quality for prototypical arguments reported above ( $c f$. hypothesis $1 \mathrm{~b}$ ). The absence of a similar effect in the 
supportive norm conditions might have been due to the fact that subjects tended to be opposed to the use of entrance exams, as a consequence of which subjects' reactions to the pro arguments presumably tended to be overall somewhat negative.

Under conditions of systematic message processing cognitive responses are assumed to be predictive of attitude change. The more favourable to the message are the responses, the more likely attitude change is to occur. In this sense the relationship between the valence of cognitive responses and attitudes is indicative of message elaboration. To investigate possible processing differences, regression analyses were performed with pretest attitudes and the evaluations of pro and contra arguments as predictors of post-test attitudes. When the source group norm was supportive the evaluation of the pro arguments was predictive of attitudes $(\beta=0.36$, $p<0.01$ ), while the evaluation of the contra arguments was not. This would suggest systematic processing of prototypical pro arguments ( $c f$. hypothesis 2 ). When the source norm was opposing, the evaluations of neither pro nor contra arguments were predictive of attitudes. Probably prototypical contra arguments were more readily accepted, since subjects tended to be opposed to entrance exams themselves.

\section{Argument recall}

Argument recall was rated by independent judges, who agreed in 91 per cent of the cases on the number of correctly recalled contra arguments and in 85 per cent of the cases on the number of correctly recalled pro arguments. Mean scores for the two judges (ranging from $0-3$ correctly recalled arguments) were analysed. Overall recall of contra arguments $(M=1.53)$ was higher than recall of pro arguments $(M=1.26, F(1,87)=6.35, p<0.02)$. This higher recall of contra arguments was qualified by a significant source norm by position interaction $(F(1,87)=30.22, p<0.0001)$. Contra arguments $(M=1.78)$ were better recalled than pro arguments $(M=0.93$, $t(46)=6.23, p<0.0001)$ when the source norm was opposing the use of entrance exams, i.e. when contra arguments were prototypical, while the reverse tended to be true when the source group norm was supportive of entrance exams, i.e. when pro arguments were prototypical (contra: $M=1.28$; pro: $M=1.59, t(47)=-1.85$, $p<0.07$ ). Apparently, recall is better for prototypical arguments. This would suggest more elaboration of prototypical arguments, as predicted in hypothesis 2 .

\section{EXPERIMENT 2}

The results of Experiment 1 clearly support the notion that one is inclined to conform to ingroup norms. Moreover, prototypical arguments were perceived to be of higher quality than a-prototypical arguments, as was contended by Turner et al. (1989). In accordance with the proposition that one is motivated to conform to ingroup norms (e.g. Turner et al., 1987; Abrams and Hogg, 1990) it could be argued that arguments will be perceived to be of higher quality when they may be considered to be prototypical of ingroup norms. Since subjects were only exposed to ingroup arguments in Experiment 1, it was not possible to test the proposed influence of social categorization on conformity and the perceptions of argument quality. To address this issue the source of the arguments (in- or outgroup) was varied in Exper- 
iment 2. After exposure to ingroup arguments, conformity to the ingroup norm was expected (hypothesis 1a). Moreover, prototypical ingroup arguments were predicted to be perceived as being of higher quality (hypothesis $1 \mathrm{~b}$ ).

Although indications of higher elaboration of prototypical arguments were found in the results for argument recall and the relationship between valence of cognitive responses and attitudes, results for attitudes and the number of cognitive responses were not indicative of differential processing. The absence of effects of argument quality on attitudes may in part have been due to the fact that subjects were exposed to both pro and contra arguments, which might have rendered argument quality effects somewhat diffuse. Therefore, subjects in Experiment 2 were exposed to onesided messages, i.e. either to pro or contra arguments. In accordance with the studies by Mackie et al. (1990) and Van Knippenberg and Wilke (1991) more elaboration of ingroup arguments was expected (hypothesis 2). Moreover, this more extensive elaboration was predicted to be most notable when the presented arguments were considered to be ingroup prototypical (hypothesis 3 ).

Furthermore, an attenuated manipulation of argument prototypicality was employed, since it was feared that simply labelling the presented set 'representative' or 'not representative' might be insufficient when the contrast provided by two-sided exposure was absent. In Experiment 2 the position advocated by the arguments was varied, while the alleged source group norm concerning the issue under scrutiny was explicitly stated. The combination of the position advocated by the arguments and the source group norm rendered the arguments either prototypical or a-prototypical of the source group.

\section{Method}

\section{Overview and design}

Subjects were confronted with arguments concerning the use of university entrance exams, which were allegedly generated by their own group (fellow students) or an outgroup (employees of the Ministry of Transport and Public Works). Quality of and position advocated by the presented arguments were varied over conditions. The opinion held by most members of the source group (either supporting or opposing the use of entrance exams) was explicitly stated before message presentation. This yielded a 2(source: ingroup/outgroup) $\times 2$ (source norm: supporting/opposing) $\times$ 2 (argument quality: strong/weak) $\times 2$ (position advocated by arguments: pro/contra) between-subjects design. Information about the source norm together with the advocated argument position denoted arguments as either prototypical of the source opinion or not. The main dependent variables were attitudes assessed on pre- and post-test, cognitive responses to the message and argument recall.

\section{Subjects}

One hundred and thirty-nine law undergraduates (53 male, 86 female) participated in the experiment, receiving Dfl. 10 for their cooperation. Subjects were randomly assigned to experimental conditions. No initial differences in attitudes towards entrance exams, as measured on the pretest, were found $(M=14.28)$. 


\section{Procedure}

Procedures closely resembled those of the first study. Attitudes towards the use of university entrance exams were assessed together with the request to volunteer in the experiment (pretest). This pretest allegedly took place not only among law undergraduates, but also among employees of the Ministry of Transport and Public Works (TPW). The TPW was considered to be an outgroup of approximately equal status, with no vested interest in the issue (vested interest might result in discounting of outgroup arguments, $c f$. Eagly and Chaiken, 1984) ${ }^{1}$.

Following procedures similar to those of the first study, subjects were exposed to a set of either pro of contra arguments supposedly originating from in-or outgroup. The argument sets were identical to those used in the first study. Before reading the arguments, subjects were informed about the attitudes towards the use of entrance exams allegedly held by most of the source's members (experimentally varied as either supporting or opposing entrance exams). Furthermore, the position advocated by the arguments in the set was announced in advance, followed by the statement that this subset of arguments could be assumed to be either representative or nonrepresentative of the source group opinion. When the source group norm supported the use of entrance exams, pro arguments were considered to be prototypical and contra arguments were considered a-prototypical, while the reverse was true when the source group norm opposed the use of entrance exams.

After reading the arguments, subjects again expressed their attitudes towards entrance exams (post-test), completed a thought-listing task and rated argument quality and prototypicality, source group norm and source group status and expertise (to control for possible confounding of the source group manipulation). At the end of the experiment argument recall was assessed, after which subjects were debriefed and paid for their participation.

\section{Results and discussion}

\section{Source and argument perceptions}

Perceptions of the source group norm towards the use of entrance exams were in accordance with the experimental induction (opposing: $M=12.43$; supporting: $M=20.06, F(1,123)=52.28, p<0.0001)$. The manipulation of the prototypicality of arguments appeared to be successful. When the induced source group norm supported entrance exams, pro arguments were considered to be more prototypical $(M=4.54)$ than contra arguments $(M=3.53, F(1,123)=18.68, p<0.0001)$, while pro arguments were considered to be less prototypical $(M=3.65)$ than contra arguments $(M=4.74)$ when the norm opposed the use of entrance exams $(F(1,123)=20.47$, $p<0.0001)$. Analysis of the perceived quality of arguments yielded only a significant result for the expected main effect of argument quality $(F(1,123)=14.31, p<0.0001)$.

\footnotetext{
'It might be argued that the relevance of the topic under scrutiny is higher for ingroup than for outgroup. However, relevance to ingroup is lower than one would expect at first sight, since the attitude measure concerns the use of university entrance exams, while subjects themselves had already been admitted to university.

An anonymous reviewer suggested that pro or contra messages from an outgroup source might be discounted on the basis of presumed source group characteristics. Although this possibility cannot be ruled out completely, pretesting showed subjects to have very little expectation about the outgroup position.
} 
Strong arguments were judged to be of higher quality $(M=4.58)$ than weak arguments $(M=3.78)$. Note that the predicted higher quality ratings for prototypical ingroup arguments (hypothesis $1 b$ ) were not apparent. No differences in perceived source status or expertise were found.

\section{Attitudes}

Post-test attitude scores were analysed by means of ANCOVA with pretest scores as the covariate $(F(1,122)=126.71, p<0.0001$ for the regression). A summary table of means (adjusted for the pretest) is displayed in Table 2. Subjects appeared to conform to the normative ingroup position, as indicated by the predicted source of norm interaction (hypothesis 1a, $F(1,122)=6.89, p<0.01$ ). After exposure to ingroup arguments, subjects' attitudes were affected by the source norm, an opposing norm resulting in opposing attitudes $(M=13.36)$, while a supporting norm elicited slightly supportive attitudes $(M=16.98, F(1,122)=7.95, p<0.006)$. After exposure to outgroup arguments, attitudes were unaffected by the source norm (contra: $M=14.05$; pro: $M=13.63$ ). This result supports the notion that one's motivation to conform to group norms is mediated by social categorization processes.

Subjects' attitudes turned out to be affected by the position advocated by the arguments, with contra arguments eliciting more opposing attitudes $(M=13.11)$ than pro arguments $(M=15.39, F(1,122)=5.70, p<0.02)$. None of the tests of the effects of argument quality reached significance, i.e. no indication of higher elaboration of (prototypical) ingroup arguments (hypotheses 2 and 3) was found. This issue will be addressed below.

Table 2. Post-test attitudes, adjusted for the pretest, Experiment 2

\begin{tabular}{|c|c|c|c|c|}
\hline \multirow[b]{3}{*}{ Position } & \multicolumn{4}{|c|}{ Source norm } \\
\hline & \multicolumn{2}{|c|}{ Supporting } & \multicolumn{2}{|c|}{ Opposing } \\
\hline & Pro & Contra & Pro & Contra \\
\hline \multicolumn{5}{|l|}{ Ingroup } \\
\hline Strong & 17.90 & 18.05 & 16.09 & 10.38 \\
\hline Weak & 15.73 & 16.25 & 15.59 & 11.37 \\
\hline \multicolumn{5}{|l|}{ Outgroup } \\
\hline Strong & 14.24 & 11.12 & 14.29 & 12.83 \\
\hline Weak & 13.33 & 11.82 & 15.99 & 13.08 \\
\hline
\end{tabular}

\section{Cognitive responses}

The cognitive responses assessed by the thought-listing task were categorized by two independent judges, rating target of the responses (issue-relevant, source-related or irrelevant, 99 per cent agreement) and evaluation expressed by the responses (positive, negative or neutral, 75 per cent agreement). Means scores for the two judges were analysed.

Analysis of the number of issue-relevant cognitive responses yielded a significant 
source by quality interaction $(F(1,123)=5.43, p<0.03)$. More issue-relevant responses were observed after exposure to strong arguments generated by the ingroup $(M=3.33)$ than after exposure to strong arguments assumed to originate from an outgroup $(M=2.77, F(1,123)=4.75, p<0.04)$, while the reverse tended to be true after exposure to weak arguments (ingroup, weak: $M=2.96$; outgroup, weak: $M=3.23, F(1,123)=1.13$, n.s. $)$. This would seem to indicate that strong ingroup arguments and weak outgroup arguments elicited more extensive elaboration. The number of source-related responses was considered to be too small for a meaningful analysis.

Analysis of the valence of the cognitive responses revealed, as expected, a significant effect of argument quality $(F(1,123)=9.41, p<0.003)$. Strong arguments $(M=0.31)$ elicited more positive evaluations than weak arguments $(M=-0.64)$. Furthermore, more positive evaluations were observed after exposure to ingroup arguments $(M=0.12)$ than after exposure to outgroup arguments $(M=-0.49, F(1,123)=3.90$, $p<0.05)$ and contra arguments resulted in more positive responses $(M=0.16)$ than pro arguments $(M=-0.51, F(1,123)=4.97, p<0.03)$. These effects were qualified by the source by position interaction $(F(1,123)=6.05, p<0.02)$. It appeared that pro arguments resulted in more negative responses $(M=-1.23)$ than contra arguments $(M=0.22, F(1,123)=10.54, p<0.002)$, when they were believed to originate from an outgroup, but not when they were believed to originate from the ingroup (ingroup, pro: $M=0.14$; ingroup, contra: $M=0.10, F<1$ ). The negative evaluations elicited by pro arguments originating from an outgroup may be due to the circumstance that they were both counter-attitudinal (subjects tended to be opposed to the use of entrance exams) and generated by an outgroup. This could indicate some bias in the judgement of arguments.

Although the source by quality interaction did not reach significance $(F(1,123)=1.32$, n.s. $)$, means for this interaction display an interesting pattern. Strong ingroup arguments $(M=0.76)$ elicited more positive cognitive responses than weak ingroup arguments $(M=-0.53, F(1,123)=9.14, p<0.003)$, while the evaluation of strong $(M=-0.20)$ and weak $(M=-0.76)$ outgroup arguments did not differ significantly $(F(1,123)=1.72$, n.s.). This could indicate more elaboration of the ingroup message ( $c f$. hypothesis 2 ). Strong arguments are expected to result in more positive cognitive responses than weak arguments only when arguments are processed systematically ( $c f$. Petty and Cacioppo, 1981, 1986). Viewed from a different angle, an alternative explanation presents itself. While no differences in message evaluation between in- and outgroup were found for weak arguments $(F<1)$, strong ingroup arguments were evaluated more positively than strong outgroup arguments $(F(1,123)=5.40, p<0.03)$. The fact that strong arguments are judged more positively when they originate from ingroup than when they originate from outgroup may be viewed as an instance of ingroup bias (more generally described by e.g. Tajfel, 1978). When arguments are weak no bias is apparent.

As in the first study, regression analyses were performed to investigate the relationship between valence of cognitive responses and attitudes. Since analysing pro and contra argument conditions separately would yield rather low $N$ 's, attitudes were recoded so that pro and contra conditions could be combined. Evaluations were only predictive of attitudes when subjects were exposed to prototypical ingroup arguments $(\beta=0.34, p<0.02)$, which suggests that only prototypical ingroup arguments were processed systematically ( $c f$. hypothesis 3 ). 


\section{Argument recall}

The number of arguments correctly recalled was rated by independent judges, who agreed in 82 per cent of the cases. Mean scores for the two judges were analysed.

Testing yielded a significant source by quality interaction $(F(1,123)=4.78$, $p<0.03)$. Strong outgroup arguments $(M=1.88)$ resulted in less recall than weak outgroup arguments $(M=2.27, F(1,123)=5.20, p<0.03)$, while no differences in recall for strong $(M=2.17)$ and weak ingroup arguments $(M=2.03)$ were found $(F<1)$. Two possible interpretations of this result may be suggested. Assuming there is no interest in outgroup messages, which would result in less elaboration of outgroup arguments, one would expect recall of outgroup arguments to be generally lower. When, however, the outgroup message appears to offer the opportunity to derogate the outgroup or the outgroup message, in this case when outgroup arguments are clearly weak, one may be motivated to scrutinize the outgroup message, resulting in higher recall of weak outgroup arguments. The means for the number of issuerelevant cognitive responses for the source by quality interaction (more responses after exposure to strong ingroup or weak outgroup arguments) seem to support this view. An alternative interpretation of this interaction effect is that it is not caused by processing differences, but rather by a bias in message recall. Arguments offering the opportunity to reach or maintain a negative judgement of the outgroup or outgroup message (i.e. weak arguments) are preferred, and hence better recalled. Both interpretations suggest a preference for strong ingroup and weak outgroup arguments.

A probably related effect is the source by position interaction $(F(1,123)=5.04$, $p<0.03)$. Pro arguments from an ingroup source $(M=1.96)$ and contra arguments from an outgroup source $(M=1.97)$ tended to result in less recall than contra arguments from an ingroup source $(M=2.24)$ and pro arguments from an outgroup source $(M=2.20)$. Means did, however, not differ significantly. A possible interpretation of this interaction is that one wishes to maintain the idea that one agrees with the ingroup or disagrees with the outgroup (subjects tended to be opposed to the use of entrance exams, and would hence tend to agree with the position advocated by the contra arguments). This interpretation would again suggest the presence of biases in message recall. Recall appears to be better for information supporting the notion that one is similar to ingroup or dissimilar to outgroup. Results from a different area of research show that recall is better for information consistent with attitudinal preferences (Roberts, 1985). The recall effects reported here might be indicative of a similar bias.

\section{GENERAL DISCUSSION}

The results reported above offer support for the hypothesized tendency to conform to ingroup norms. After exposure to an ingroup message and information about ingroup attitudes, subjects appeared to conform to the source group norm, while this was not the case after exposure to outgroup arguments. This differential effect supports the self-categorizational contention of the mediating role social categorization will play in inducing attitude change. Although prototypical arguments were perceived to be of higher quality in Experiment 1, the predicted higher quality ratings 
for prototypical ingroup arguments were not observed in Experiment 2. This issue will be addressed after the discussion of the processing effects below.

Results for the analysis of the relation between message elaboration and argument prototypicality seem to be somewhat equivocal. Although in the first study results for argument recall and the cognitive response-attitude relation seemed to be supportive of the notion that prototypical arguments were processed more systematically, results for attitudes and number of cognitive responses were not indicative of differential processing. In the second study cognitive responses were only predictive of attitudes after exposure to prototypical ingroup arguments, suggesting systematic processing. However, no further evidence of differential processing of prototypical ingroup arguments was found. On the whole, message elaboration appeared to be rather low. This may in part have been due to the specific manipulation of argument prototypicality, which bore a direct relation to attitudes. In Experiment 2 the source opinion was explicitly stated in advance. This information might have functioned as a simple persuasion cue ( $c f$. Chaiken, 1980, 1987), offering subjects the possibility to gather the preferred information (the source opinion) without engaging in extensive message elaboration. In the absence of such information, prototypical messages may indeed elicit systematic processing ${ }^{2}$. This notion appears, however, to require further investigation.

The fact that in the second study prototypicality did not affect ratings of (ingroup) argument quality might be ascribed to the presumably low degree of message elaboration. Possibly a minimal degree of elaboration is needed for prototypicality to affect perceptions of argument quality ${ }^{3}$. Again, further testing seems in order before firm conclusions may be drawn here.

The present studies may be related to a different body of research when they are reformulated as pertaining to minority/majority influence. Prototypical arguments may be argued to represent the majority's point of view, while a-prototypical arguments would represent a minority. Persuasion based on minority communication is contended to involve more cognitive effort than majority-induced persuasion (e.g. Moscovici, 1980; Nemeth, 1986; see Maass and Clark, 1984, for a review). Contrary to this assertion, the present results would appear to indicate more scrutinizing of majority than minority arguments. This apparent contradiction might be attributed to the specific experimental framing. A majority-minority categorization is not necessarily implicated: any group member may hold both prototypical and a-prototypical arguments. However, although no conclusions concerning minority-majority influence should be based on the present study, taking other evidence for higher elaboration of majority communications into consideration (Mackie, 1987), it might be better to replace questions about whether or not minority influence is based on more cognitive effort with questions about when minorities and majorities are likely to elicit systematic processing or the conditions under which minority and majority influence processes will and will not differ (cf. Chaiken and Stangor, 1987).

\footnotetext{
${ }^{2}$ As was noted by an anonymous reviewer, the attitude topic appears not to be group definitional, i.e. it is not particularly ingroup relevant, which may have rendered the motivation to elaborate rather low as compared to a situation in which a more ingroup-relevant topic is under scrutiny. I.e. the choice of topic may have rendered systematic processing somewhat less likely.

${ }^{3}$ As was noted by an anonymous reviewer, the possibility that perceptions of prototypical argument quality were a consequence rather than an antecedent of position acceptance cannot be ruled out. Although group norms affected both attitudes and perceived argument quality, this does not necessarily mean that group norms affected attitudes through perceived higher quality of prototypical arguments.
} 
It was proposed that the results for the analysis of the cognitive responses and argument recall in experiment 2 suggested an intergroup bias in message processing and recall. Ingroup arguments elicited more positive responses (when counter-attitudinal) than outgroup arguments, and recall appeared to be better for arguments supporting the view that the outgroup's position is ill-founded and that one agrees with ingroup of disagrees with outgroup. Results of a study by Mackie, GastardoConaco and Skelly (1990) seem to suggest a similar bias in argument recall. Mackie et al. presented subjects with both position-congruent and -incongruent arguments from either an in- or outgroup member. Position-congruent ingroup arguments resulted in higher recall than position-incongruent ingroup arguments, while the reverse was true for outgroup arguments. Furthermore, strong position-congruent ingroup arguments resulted in higher recall than weak arguments, while again the reverse pattern was found for outgroup arguments. This seems to suggest a preference for arguments supporting the view that the ingroup is consistent, while the outgroup is inconsistent, and that the ingroup position is well-founded, while the outgroup position is ill-founded. The recall biases in the study by Mackie et al. appeared to be clearest when the source position was known beforehand, i.e. when message elaboration may be assumed to be low. As was argued above, message elaboration appeared to be rather low in the second study presented here. It could be argued that the processing and recall biases reported here will be more likely to appear under conditions of low elaboration. When message elaboration is low one apparently employs simple decision rules ( $c f$. Chaiken, 1980, 1987) suggesting higher validity of ingroup norms and ingroup messages. Although it is not possible to test for this possibility in the present study, the results of the study by Mackie et al. seem to corroborate this explanation.

\section{REFERENCES}

Abrams, D. and Hogg, M. A. (1990). 'Social identification, self-categorization and social influence'. In: Doise, W. and Hewstone, M. (Eds) European Review of Social Psychology, Vol. 1, John Wiley \& Sons, Chichester.

Abrams, D., Wetherell, M., Cochrane, S., Hogg, M. A. and Turner, J. C. (1990). 'Knowing what to think by knowing who you are: Self-categorization and the nature of norm formation, conformity and group polarization', British Journal of Social Psychology, 29: 97-119.

Chaiken, S. (1980). 'Heuristic versus systematic information processing and the use of source versus message cues in persuasion', Journal of Personality and Social Psychology, 39: 752-766.

Chaiken, S. (1987). 'The heuristic model of persuasion'. In: Zanna, M. P., Olsen, J. M. and Herman, C. P. (Eds) Social Influence: The Ontario Symposium, Vol. 5, Erlbaum, Hillsdale, NJ.

Chaiken, S. and Stangor, C. (1987). 'Attitudes and attitude change', Annual Review of Psychology, 38: 575-630.

Deutsch, M. and Gerard, H. B. (1955). 'A study of normative and informational influences upon individual judgment', Journal of Abnormal and Social Psychology, 51: 629-636.

Eagly, A. H. and Chaiken, S. (1984). 'Cognitive theories of persuasion'. In: Berkowitz, L. (Ed.) Advances in Experimental Social Psychology, Vol. 17, Academic Press, New York.

Festinger, L., Schachter, S. and Back, K. (1950). Social Pressures in Informal Groups, Harper \& Row, New York.

Hogg, M. A. and Turner, J. C. (1987). 'Social identity and conformity: A theory of referent informational influence'. In: Doise, W. and Moscovici, S. (Eds) Current Issues in European Social Psychology, Vol. 2, Cambridge University Press.

Kiesler, C. A. and Kiesler, S. B. (1969). Conformity, Addison-Wesley, Reading, MA. 
Maass, A. and Clark, R. D., III (1984). 'Hidden impact of minorities: Fifteen years of minority influence research', Psychological Bulletin, 95: 428-450.

Mackie, D. M. (1986). 'Social identification effects in group polarization', Journal of Personality and Social Psychology, 50: 720-728.

Mackie, D. M. (1987). 'Systematic and nonsystematic processing of majority and minority persuasive communications', Journal of Personality and Social Psychology, 53: 41-52.

Mackie, D. M. and Cooper, J. (1984). 'Attitude polarization: Effects of group membership', Journal of Personality and Social Psychology, 46: 575 585.

Mackie, D. M., Worth, L. T. and Asuncion, A. G. (1990). 'Processing of persuasive in-group messages', Journal of Personality and Social Psychology, 58: 812-822.

Mackie, D. M., Gastardo-Conaco, M. C. and Skelly, J. J. (1990). 'Knowledge of the advocated position and the processing of in-group and out-group persuasive messages'. Manuscript submitted for publication.

Moscovici, S. (1980). 'Toward a theory of conversion behavior'. In: Berkowitz, L. (Ed.) Advances in Experimental Social Psychology, Vol. 13, Academic Press, New York, pp. 209_ 239.

Nemeth, C. J. (1986). 'Differential contributions of majority and minority influence', Psychological Review, 93: 23-32.

Petty, R. E. and Cacioppo, J. T. (1981). Attitudes and Persuasion: Classic and Contemporary Approaches, Wm. C. Brown, Dubuque, Iowa.

Petty, R. E. and Cacioppo, J. T. (1986). 'The elaboration likelihood model of persuasion'. In: Berkowitz, L. (Ed.) Advances in Experimental Social Psychology, Vol. 19, Academic Press, New York.

Roberts, J. V. (1985). 'The attitude-memory relationship after 40 years: A meta-analysis of the literature', Basic and Applied Social Psychology, 6: 221-241.

Sherif, M. (1936). The psychology of social norms, New York: Harper \& Bros.

Tajfel, H. (1978). Differentiation Between Social Groups. Studies in the Social Psychology of Intergroup Relations, Academic Press, London.

Turner, J. C., Hogg, M. A., Oakes, P. J., Reicher, S. D. and Wetherell, M. S. (1987). Rediscovering the Social Group. A Self-Categorization Theory, Basil Blackwell, Oxford.

Turner, J. C., Wetherell, M. S. and Hogg, M. A. (1989). 'Referent informational influence and group polarization', British Journal of Social Psychology, 28: 135-147.

Van Knippenberg, D. and Wilke, H. (1991). 'Sociale categorisatie, verwerking van argumenten en attitudeverandering'. In: van der Pligt, J., van der Kloot, W., van Knippenberg, A. and Poppe, M. (Eds) Fundamentele Sociale Psychologie, Vol. 5, Tilburg University Press.

Wilder, D. A. (1990). 'Some determinants of the persuasive power of in-group and out-groups: Organization of information and attribution of independence', Journal of Personality and Social Psychology, 59: 1202-1213. 